\title{
The effects of serum granulin levels on anthropometric measures and glucose metabolism in infertile women with different ovarian reserve status
}

\author{
Ozgur Kan, Umit Gorkem \\ Department of Obstetrics and Gynaecology, Hitit University Faculty of Medicine, Corum, Turkey
}

\begin{abstract}
Introduction: Granulin (GRN) is an adipokine with proinflammatory features, which plays important role in glucose metabolism and insulin resistance pathogenesis. It has been reported that granulin precursors were localised in developing follicles in animal studies. The purpose of this study was to evaluate the association of granulin levels with anthropometric features, glucose metabolism, and ovarian reserve. Material and methods: A total of 109 infertile women were included in this cross-sectional, prospective study, who attended a tertiary clinic. All participants were categorised into diminished ovarian reserve (DOR) and normal ovarian reserve groups (NOR), in accordance with Bologna criteria. The demographic characteristics, including age, BMI, waist-hip circumferences, and biochemical parameters, were recorded. Serum granulin level was determined by enzyme-linked immunosorbent assay.

Results: No significant difference was observed in the GRN levels $(p=0.229)$ between the groups. There was a positive correlation between GRN levels and BMI, WC, HC, and 75 g oral glucose tolerance values in NOR group $(p<0.01, p<0.05, p<0.01$, and $p<0.05$, respectively). Conclusions: Our results suggest that granulin is associated with anthropometric features in infertile patients and might be an important indicator of obesity and impaired glucose metabolism. Elevated levels of granulin may have a diabetogenic effect and predispose women to high glucose levels. (Endokrynol Pol 2019; 70 (3): 255-259)
\end{abstract}

Key words: granulin; anthropometry; glucose metabolism; ovarian reserve; obesity

\section{Introduction}

Obesity and excessive body weight may affect reproductive health with different mechanisms. In numerous studies it was demonstrated that obesity is associated with poor in vitro fertilisation (IVF) outcomes [1]. It also had a relationship with dyslipidaemia and insulin resistance, as important components of the metabolic syndrome [2]. Furthermore, all of these factors were related with low-grade chronic inflammation process and can lead to cardiovascular diseases [3]. In a recent study, the authors reported that cardiovascular risk markers were increased in patients with diminished ovarian reserve [4]. Decreased oestrogen production due to loss of ovarian functions may increase dyslipidaemia and contribute to adverse effects in glucose and insulin metabolism, body fat distribution, and dysfunction in vascular endothelium [5].

Granulin (GRN), also known as acrogranin and proepithelin, is an adipokine with proinflammatory features. It is thought that elevation of circulating levels may be an important indicator in the development of chronic inflammatory process seen in type 2 diabetes and obesity [6]. It has complex physiological and pathological functions in various conditions including wound healing, tissue remodelling, progression of cell cycle, breast and gynaecological malignancies, and neurodegenerative disorders $[7,8]$. In several animal studies it was reported that this glycoprotein possessed oocyte-specific expression, and its precursors were localised in granulosa cells in developing follicles [9]. In a recent study the authors stated that elevated progranulin levels are associated with obesity in infertile women, and progranulin may be a promising therapeutic target to prevent obesity and infertility [10].

As an important adipokine with proinflammatory features and oocyte-specific expression in follicles, we hypothesise that GRN may be associated with glucose metabolism and ovarian reserve in infertile patients. The purpose of this study was to evaluate the levels of granulin in non-polycystic infertile patients and to analyse the relationship between GRN and anthropometric features, glucose metabolism, and ovarian reserve status. 


\section{Material and methods}

The cross-sectional study was planned to be carried out at the Hitit University Reproductive Health Centre between January 2016 and September 2016. This study was approved by the Ethics Committee of Ankara Numune Training and Research Hospital and conducted in accordance with the institutional guidelines and Helsinki declaration (reference number: E-15-563). Informed consent was obtained from each of the participants immediately prior to the study.

A total of 109 women seeking fertility assistance were enrolled in this prospective study. In the first step, women with any foreknown systemic chronic diseases (diabetes mellitus, autoimmune and inflammatory diseases), history of pelvic surgery, chemoradiotherapy due to malignancies, current smoking, and drug use that may affect ovarian reserve were excluded. Subsequently, women with PCOS, which may affect anthropometric features and glucose metabolism, were not included in the study. The diagnosis of PCOS was based on the recent Amsterdam ESHRE/ ASRM proposal [11].

The participant women who met the inclusion criteria were divided into two groups according to their ovarian status, as normal ovarian reserve (NOR) and diminished ovarian reserve (DOR) groups. The diagnosis of DOR was based on the Bologna criteria of the ESHRE consensus [12]. At least two of the following three characteristics were needed to diagnose the patient as DOR; (i) advanced maternal age ( $\geq 40$ years) or any other risk factor for poor ovarian response; (ii) a previous poor response history $(\leq 3$ oocytes with a conventional stimulation protocol); (iii) an abnormal ovarian reserve test (i.e. antral follicle counting $<5-7$ follicles or anti-Mullerian hormone levels $<0.5-1.1 \mathrm{ng} / \mathrm{dL}$ ).

First, all anthropometric measurements including height, weight, waist circumference (WC), hip circumferences (HC), and waist-hip circumference ratio (WHR) were performed by the same observer using the same method with the same scale. Body mass index (BMI) was calculated as weight $(\mathrm{kg}) / \mathrm{height}^{2}\left(\mathrm{~m}^{2}\right)$. Both groups were compared in terms of their anthropometric features.

For assessment of serum follicle stimulating hormone (FSH), luteinising hormone (LH), oestradiol (E2), anti-Mullerian hormone (AMH) and granulin levels, venous blood samples $(5 \mathrm{ml})$ were collected from the participants in the morning, after an approximately eight-hour fasting period on their early follicular phase. Following collection, samples were centrifuged at $3000 \mathrm{rpm}$ for 20 minutes, and separated plasma for $\mathrm{AMH}$ and GRN were stored at $-80^{\circ} \mathrm{C}$ until analysis. The samples were analysed on a daily basis for E2, FSH, and LH using electrochemiluminescence immunoassay (ECLIA) method with an auto-analyser (Cobas 6000, E 601 Roche Diagnostics, GmbH, Mannheim, Germany). AMH samples were measured by the same method with an auto-analyser (Cobas 6000, E 601 Roche Diagnostics, $\mathrm{GmbH}$, Mannheim, Germany). The GRN levels were measured using enzyme-linked immunosorbent assay (ELISA) method and granulin assay kits (Biotek Synergy HT, Cloud-Clone Corp., Houston, USA). Comparisons and correlation analyses of all data were performed.

\section{Statistical analyses}

All data analyses were performed using SPSS (Statistical Packages for the Social Sciences) software, version 22 (SPSS Inc., Chicago, USA). The Kolmogorov-Smirnov and Shapiro-Wilk tests were used to test normality of distribution. Continuous parameters were presented as mean \pm standard deviation (SD). Continuous variables were compared using independent samples t-test due to normally distributed data. Pearson or Spearman correlation analysis, where appropriate, was used to test whether granulin showed any significant linear relationship with other study parameters. A $p$ value less than 0.05 was considered as statistically significant.

\section{Results}

Eighty-four women with NOR and 25 women with DOR were included in this study. The demographic and anthropometric characteristics are shown in Table I. As expected, the mean age of the DOR group was higher than the mean age of the control group (37.4 vs. 30.0, $\mathrm{p}<0.001)$. There was no significant difference between the two groups for the anthropometric features, including BMI, WC, HC, and WHR ( $p>0.05$, for all). While serum E2 and LH levels were comparable between the groups, higher FSH and lower AMH levels were observed in the DOR group (7.21 \pm 1.89 vs. $9.15 \pm 2.68$ and $2.93 \pm 1.85$ vs. $1.38 \pm 2.12, \mathrm{p}<0.001$, respectively). No significant difference was observed between the GRN levels $(\mathrm{p}=0.229)$ in both groups.

Table I. Baseline anthropometric and biochemical characteristics of the participant women

\begin{tabular}{|c|c|c|c|}
\hline & DOR group $(n=25)$ & NOR group $(n=84)$ & $\mathbf{p}$ \\
\hline Age [years] & $37.4 \pm 3.4$ & $30.0 \pm 5.7$ & $0.000 * *$ \\
\hline BMI $\left[\mathrm{kg} / \mathrm{m}^{2}\right]$ & $25.5 \pm 4.3$ & $26.6 \pm 5.1$ & 0.443 \\
\hline$W C[\mathrm{~cm}]$ & $89.6 \pm 9.1$ & $89.8 \pm 11.7$ & 0.947 \\
\hline $\mathrm{HC}[\mathrm{cm}]$ & $105.1 \pm 6.7$ & $105.4 \pm 10.4$ & 0.878 \\
\hline WHR & $0.9 \pm 0.1$ & $0.9 \pm 0.1$ & 0.789 \\
\hline $\mathrm{FSH}[\mathrm{IU} / \mathrm{mL}]$ & $9.2 \pm 2.7$ & $7.2 \pm 1.9$ & $0.001 * *$ \\
\hline $\mathrm{LH}[\mathrm{IU} / \mathrm{mL}]$ & $6.7 \pm 3.0$ & $5.8 \pm 2.1$ & 0.240 \\
\hline $\mathrm{E}_{2}[\mathrm{pg} / \mathrm{mL}]$ & $47.7 \pm 25.8$ & $46.8 \pm 26.1$ & 0.957 \\
\hline $\mathrm{AMH}[\mathrm{ng} / \mathrm{mL}]$ & $1.4 \pm 2.1$ & $2.9 \pm 1.9$ & $0.000 * *$ \\
\hline Fasting glucose [mg/dL] & $99.3 \pm 39.9$ & $91.8 \pm 12.2$ & 0.773 \\
\hline 75-g OGTT [mg/dL] & $129.1 \pm 79.3$ & $105.1 \pm 32.4$ & 0.119 \\
\hline Granulin [ng/mL] & $3.1 \pm 1.2$ & $2.7 \pm 1.1$ & 0.141 \\
\hline
\end{tabular}

DOR — diminished ovarian reserve; NOR — normal ovarian reserve; BMI — body mass index; WC — waist circumference; HC — hip circumference;

WHR — waist-hip ratio; FSH — follicle-stimulating hormone; $\mathrm{LH}$ — luteinising hormone; $\mathrm{E}_{2}$ — oestradiol; AMH — anti-Mullerian hormone; OGTT — oral glucose tolerance test. Values are expressed as mean $\pm \mathrm{SD} ;{ }^{* *} \mathrm{p}<0.01$ 
Table II. Correlation analysis of granulin with other parameters according to ovarian reserve status

\begin{tabular}{lcc}
\hline & \multicolumn{2}{c}{ Granulin levels } \\
\cline { 2 - 3 } & DOR group & NOR group \\
\hline Age $[$ years] & 0.301 & 0.033 \\
\hline BMl $\left[\mathrm{kg} / \mathrm{m}^{2}\right]$ & 0.066 & $\mathbf{0 . 2 8 8 ^ { * * }}$ \\
\hline WC $[\mathrm{cm}]$ & 0.119 & $\mathbf{0 . 3 2 4}^{* *}$ \\
\hline HC $[\mathrm{cm}]$ & -0.339 & $\mathbf{0 . 2 6 6}^{*}$ \\
\hline WHR & 0.383 & 0.206 \\
\hline FSH $[\mathrm{IU} / \mathrm{mL}]$ & 0.186 & -0.113 \\
\hline LH $[\mathrm{IU} / \mathrm{mL}]$ & 0.055 & -0.171 \\
\hline $\mathrm{E}_{2}[\mathrm{pg} / \mathrm{mL}]$ & 0.185 & -0.091 \\
\hline AMH $[\mathrm{ng} / \mathrm{mL}]$ & -0.388 & 0.053 \\
\hline $75-\mathrm{g} 0 \mathrm{GTT}[\mathrm{mg} / \mathrm{dL}]$ & 0.168 & $\mathbf{0 . 2 5 4}$ \\
\hline
\end{tabular}

DOR — diminished ovarian reserve; NOR — normal ovarian reserve; $\mathrm{BMI}$ - body mass index; WC — waist circumference; $\mathrm{HC}$ - hip circumference WHR — waist-hip ratio; FSH — follicle-stimulating hormone; $\mathrm{LH}$ — luteinising hormone; $\mathrm{E}_{2}$ — oestradiol; AMH — anti-Mullerian hormone; OGTT — oral glucose tolerance test

For each group, the relationship of GRN with other parameters were analysed with Spearman-Brown correlation, and the results are shown in Table II. There was a weak positive correlation between GRN levels and BMI, WC, and HC in the NOR group $(\mathrm{r}=0.288$; $\mathrm{p}<0.01, \mathrm{r}=0.324 ; \mathrm{p}<0.05$ and $\mathrm{r}=0.266 ; \mathrm{p}<0.01$, respectively). However, no significant correlation was found between these parameters in patients with DOR ( $\mathrm{r}=-0.066 ; \mathrm{p}>0.05, \mathrm{r}=0.119 ; \mathrm{p}>0.05$, $r=-0.339 ; p>0.05)$. In addition, there was a weak positive correlation between serum granulin concentration and $75 \mathrm{~g}$ oral glucose tolerance (OGTT) values in the NOR group $(\mathrm{r}=0.254 ; \mathrm{p}<0.05)$. However, in the DOR group, no significant correlation was found between glucose tolerance test results and GRN levels $(\mathrm{r}=0.167 ; \mathrm{p}>0.05)$.

The correlation analysis of the GRN levels with the parameters in the study independent from ovarian reserve status is shown in Table III. A significant weak positive correlation was found between GRN and BMI, WC, WHR, and 75-g OGTT ( $\mathrm{r}=0.191 ; \mathrm{p}<0.05$, $\mathrm{r}=0.257 ; \mathrm{p}<0.01, \mathrm{r}=0.220 ; \mathrm{p}<0.01, \mathrm{r}=0.228$; $\mathrm{p}<0.05$, respectively). No significant correlation was found between serum GRN level and age.

\section{Discussion}

In the present study, we focused on serum GRN concentrations, a novel adipokine with inflammatory and endocrine properties, and its association with anthropometric features, and glucose metabolism in women with non-polycystic infertile patients.
Table III. Correlation analysis of granulin levels with study parameters

\begin{tabular}{lcc}
\hline & $\mathbf{r}$ & $\mathbf{p}$ \\
\hline Age [years] & 0.154 & 0.111 \\
\hline BMI $\left[\mathrm{kg} / \mathrm{m}^{2}\right]$ & $\mathbf{0 . 1 9 1}$ & $\mathbf{0 . 0 4 7 ^ { * }}$ \\
\hline WC $[\mathrm{cm}]$ & $\mathbf{0 . 2 5 7}$ & $\mathbf{0 . 0 0 7 ^ { * * }}$ \\
\hline HC $[\mathrm{cm}]$ & 0.145 & 0.134 \\
\hline WHR & $\mathbf{0 . 2 2 0}$ & $\mathbf{0 . 0 2 2 ^ { * }}$ \\
\hline FSH $[\mathrm{IU} / \mathrm{mL}]$ & 0.021 & 0.830 \\
\hline LH $[\mathrm{IU} / \mathrm{mL}]$ & -0.076 & 0.432 \\
\hline $\mathrm{E}_{2}[\mathrm{pg} / \mathrm{mL}]$ & -0.019 & 0.848 \\
\hline AMH $[\mathrm{ng} / \mathrm{mL}]$ & -0.107 & 0.270 \\
\hline $75-\mathrm{g} 0 \mathrm{GTT}[\mathrm{mg} / \mathrm{dL}]$ & $\mathbf{0 . 2 2 8}$ & $\mathbf{0 . 0 1 7}$ \\
\hline
\end{tabular}

$\mathrm{BMI}$ - body mass index; WC — waist circumference; HC — hip circumference; WHR - waist-hip ratio; $\mathrm{FSH}$ — follicle-stimulating hormone; $\mathrm{LH}$ - luteinising hormone; $\mathrm{E}_{2}$ : — oestradiol; AMH — anti-Mullerian hormone; OGTT — oral glucose tolerance test. ${ }^{*} p<0.05,{ }^{* *} p<0.01$

Granulin is a prominent adipokine and its association with reproductive biology has been indicated in several studies. Suzuki et al. reported that GRN acted as an autocrine stimulator during the oocyte maturation process [9]. In another animal study, Vendola et al. suggested that GRN may augment early follicle development, similarly to the androgenic effect in monkeys [13]. Other studies also suggest that GRN and its precursors play a major role in the development of ovarian premature follicle [14]. Although GRN was thought to be effective on ovarian folliculogenesis and embryogenesis, we did not find a significant correlation between granulin and ovarian reserve tests. Similar to our results, Ersoy et al. also did not find any association between GRN and FSH levels in patients with premature ovarian failure (POF) [15]. The authors therefore suggested that the association of GRN with ovarian failure may be encountered as a result of POF, regardless of the impact of FSH or ovarian reserve tests.

In our study, we found a significant relationship between GRN and BMI, WHR, and glucose tolerance test regardless of ovarian reserve status. Recently, it was found that in peripheral tissues, excessive limits of GRN are closely linked to obesity and insulin resistance [16]. In another study, the authors reported that circulating GRN levels were positively correlated with body mass index and glucose levels [17]. As a result, it was thought that levels of this adipokine might increase due to uncontrolled expansion in adipose, and this condition might provoke obesity by stimulating angiogenesis and promoting growth in target tissue [16]. Although measurement of GRN levels from these studies seems useful in predicting inflammation and obesity-related metabolic diseases, further studies are 
needed to determine if measuring GRN levels will be useful as a reliable biomarker of metabolic syndrome.

Interestingly, the present results also demonstrated that serum GRN levels did not have any relationship with age, regardless of ovarian reserve status. Similar to our results, Ersoy et al. also did not find a correlation between age and serum GRN levels [15]. When the relationship between GRN levels and age was examined according to ovarian reserve status, there was a significant relationship between age and granulin in normoresponder patients. Considering that patients with normal ovarian reserve are younger than poor responders, it is thought that granulin may be involved in ovarian response physiology, especially in early age. As shown in plants, GRN levels gradually decrease with aging [18]. In light of these findings, Ersoy et al. proposed that GRN levels may be an indicator for senescence of the human ovary, rather than human life senescence [15]. However, the role of GRN in the mechanism of this topic remains unclear.

The relationship between GRN and PCOS and its possible metabolic effects has been previously evaluated in many studies. It is known that insulin resistance plays an important role in PCOS pathophysiology. In a recent study from Turkey, higher progranulin levels in women with PCOS were found compared with non-PCOS patients [19]. The authors proposed that cysteine-rich growth factors like progranulin may cause excessive early follicular growth as androgens. Zhou et al. also investigated the possible effects of progranulin in PCOS patients and found higher progranulin levels in follicular fluid [20]. These findings suggest that progranulin may be used as a marker for insulin resistance in the follicular compartment in polycystic patients.

The strengths of our study include its prospective and multivariable design, being the first to compare serum GRN levels in patients with different ovarian reserve status, and evaluating the effects of GRN on glucose metabolism and anthropometric features regardless of ovarian reserve. Our findings are limited by its relatively small sample size, and further studies with larger groups are warranted. In addition, it is possible to criticise why insulin level measurements and lipid profile-related values are not investigated in order to calculate homeostatic model assessment for the insulin resistance (HOMA-IR) index. Because these data were not investigated, the feasibility of GRN levels in predicting cardiovascular risk factors could not be investigated.

\section{Conclusions}

We demonstrated a positive correlation between serum GRN concentration and anthropometric measures in the NOR group. However, our findings did not support any correlation of GRN with these measures in women with DOR. Interestingly, there was a positive correlation between serum GRN level and 75-g OGTT only in the NOR group. In other words, it is possible to propose that elevated GRN levels may have a diabetogenic effect and predispose women to high serum glucose levels. Further large-sized prospective studies are needed to elucidate the role of GRN on glucose metabolism.

\section{Conflict of interest}

The authors declare that they have no conflict of interest.

\section{Authors' contributions}

OK and UG analysed the data. OK wrote the paper. UG conceived, designed, and performed the experiments. UG critically reviewed the final version of the article. All authors read and approved the final manuscript.

\section{Ethical approval}

This study was approved by the local Ethics Committee and the Institutional Review Board.

\section{Informed consent}

Written, informed consent was obtained from all participants.

\section{References}

1. Provost MP, Acharya KS, Acharya CR, et al. Pregnancy outcomes decline with increasing recipient body mass index: an analysis of 22,317 fresh donor/recipient cycles from the 2008-2010 Society for Assisted Reproductive Technology Clinic Outcome Reporting System registry. Fertil Steril. 2016; 105(2): 364-368, doi: 10.1016/j.fertnstert.2015.10.015, indexed in Pubmed: 26523329.

2. Farin HMF, Abbasi F, Kim SH, et al. The relationship between insulin resistance and dyslipidaemia in cigarette smokers. Diabetes Obes Metab. 2007; 9(1): 65-69, doi: 10.1111/j.1463-1326.2006.00574.x, indexed in Pubmed: 17199720.

3. Kemp DM. Does chronic low-grade endotoxemia define susceptibility of obese humans to insulin resistance via dietary effects on gut microbiota? Adipocyte. 2013; 2(3): 188-190, doi: 10.4161/adip.24776, indexed in Pubmed: 23991367.

4. Verit FF, Keskin S, Omer B, et al. Is there any relationship between cardiovascular risk markers and young women with diminished ovarian reserve? Gynecol Endocrinol. 2014; 30(10): 697-700, doi: 10.3109/09513 590.2014.922948, indexed in Pubmed: 24915163

5. Spencer CP, Godsland IF, Stevenson JC. Is there a menopausal metabolic syndrome? Gynecol Endocrinol. 1997; 11(5): 341-355, indexed in Pubmed: 9385535.

6. Youn BS, Bang SI, Klöting N, et al. Serum progranulin concentrations may be associated with macrophage infiltration into omental adipose tissue. Diabetes. 2009; 58(3): 627-636, doi: 10.2337/db08-1147, indexed in Pubmed: 19056610

7. Bateman A, Bennett HPJ. The granulin gene family: from cancer to dementia. Bioessays. 2009; 31(11): 1245-1254, doi: 10.1002/bies.200900086, indexed in Pubmed: 19795409.

8. Lu Yi, Zheng L, Zhang W, et al. Growth factor progranulin contributes to cervical cancer cell proliferation and transformation in vivo and in vitro. Gynecol Oncol. 2014; 134(2): 364-371, doi: 10.1016/j.ygyno.2014.05.025, indexed in Pubmed: 24905774.

9. Suzuki M, Matsumuro M, Hirabayashi K, et al. Oocyte-Specific Expression of Granulin Precursor (Acrogranin) in Rat Ovary. J Reprod Dev. 2000; 46: 271-277. 
10. Gorkem U, Inal ZO, Inal HA, et al. Serum progranulin levels are elevated in infertile women with obesity. Endokrynol Pol. 2018; 69(6): 661-666, doi: 10.5603/EP.a2018.0044, indexed in Pubmed: 30229554.

11. Fauser BC, Tarlatzis BC, Rebar RW, et al. Consensus on women's health aspects of polycystic ovary syndrome (PCOS): the Amsterdam ESHRE/ASRM-Sponsored 3rd PCOS Consensus Workshop Group. Fertil Steril. 2012; 97(1): 28-38, doi: 10.1016/j.fertnstert.2011.09.024, indexed in Pubmed: 22153789

12. Ferraretti AP, La Marca A, Fauser BC, et al. ESHRE working group on Poor Ovarian Response Definition. ESHRE consensus on the definition of 'poor response' to ovarian stimulation for in vitro fertilization: the Bologna criteria. Hum Reprod. 2011; 26(7): 1616-1624, doi: 10.1093/humrep/der092, indexed in Pubmed: 21505041

13. Vendola KA, Zhou J, Adesanya OO, et al. Androgens stimulate early stages of follicular growth in the primate ovary. J Clin Invest. 1998; 101(12): 2622-2629, doi: 10.1172/JCI2081, indexed in Pubmed: 9637695.

14. Hsueh AJW, Kawamura K, Cheng Y, et al. Intraovarian control of early folliculogenesis. Endocr Rev. 2015; 36(1): 1-24, doi: 10.1210/er.2015.36. issue-1.edboard, indexed in Pubmed: 25202833.

15. Ersoy AO, Oztas E, Ersoy E, et al. Granulin levels in patients with idiopathic premature ovarian failure. Eur J Obstet Gynecol Reprod Biol. 2015; 193 : 108-110, doi: 10.1016/j.ejogrb.2015.07.015, indexed in Pubmed: 26277782.
16. Matsubara T, Mita A, Minami K, et al. PGRN is a key adipokine mediating high fat diet-induced insulin resistance and obesity through IL-6 in adipose tissue. Cell Metab. 2012; 15(1): 38-50, doi: 10.1016/j.cmet.2011.12.002, indexed in Pubmed: 22225875.

17. Hossein-Nezhad A, Mirzaei K, Ansar H, et al. Obesity, inflammation and resting energy expenditure: possible mechanism of progranulin in this pathway. Minerva Endocrinol. 2012; 37(3): 255-266, indexed in Pubmed: 22766892

18. Yamada K, Matsushima R, Nishimura M, et al. A slow maturation of a cysteine protease with a granulin domain in the vacuoles of senescing Arabidopsis leaves. Plant Physiol. 2001; 127(4): 1626-1634, indexed in Pubmed: 11743107

19. Ersoy AO, Tokmak A, Ozler S, et al. Are progranulin levels associated with polycystic ovary syndrome and its possible metabolic effects in adolescents and young women? Arch Gynecol Obstet. 2016; 294(2): 403-409, doi: 10.1007/s00404-016-4096-8, indexed in Pubmed: 27071619.

20. Zhou D, Li S, Li W, et al. Increased expression of PGRN protein in follicular fluid and mRNA in granulosa cells in overweight patients with polycystic ovary syndrome. Eur J Obstet Gynecol Reprod Biol. 2017; 218: 106-112, doi: 10.1016/j.ejogrb.2017.09.017, indexed in Pubmed: 28964971. 